PROFESIONALES Y HERRAMIENTAS PARA EL DESARROLLO LOCAL Y SUS SINERGIAS TERRITORIALES. EVALUACIÓN Y PROPUESTAS DE FUTURO IX Coloquio Nacional de Desarrollo Local del GTDL-AGE 

ANTONIO MARTÍNEZ PUCHE, XAVIER AMAT MONTESINOS, ISABEL SANCHO CARBONELL y DANIEL SANCHIZ CASTAÑO (EDS.)

\section{PROFESIONALES Y HERRAMIENTAS PARA EL DESARROLLO LOCAL Y SUS SINERGIAS TERRITORIALES. EVALUACIÓN Y PROPUESTAS DE FUTURO}

IX Coloquio Nacional de Desarrollo Local del GTDL-AGE

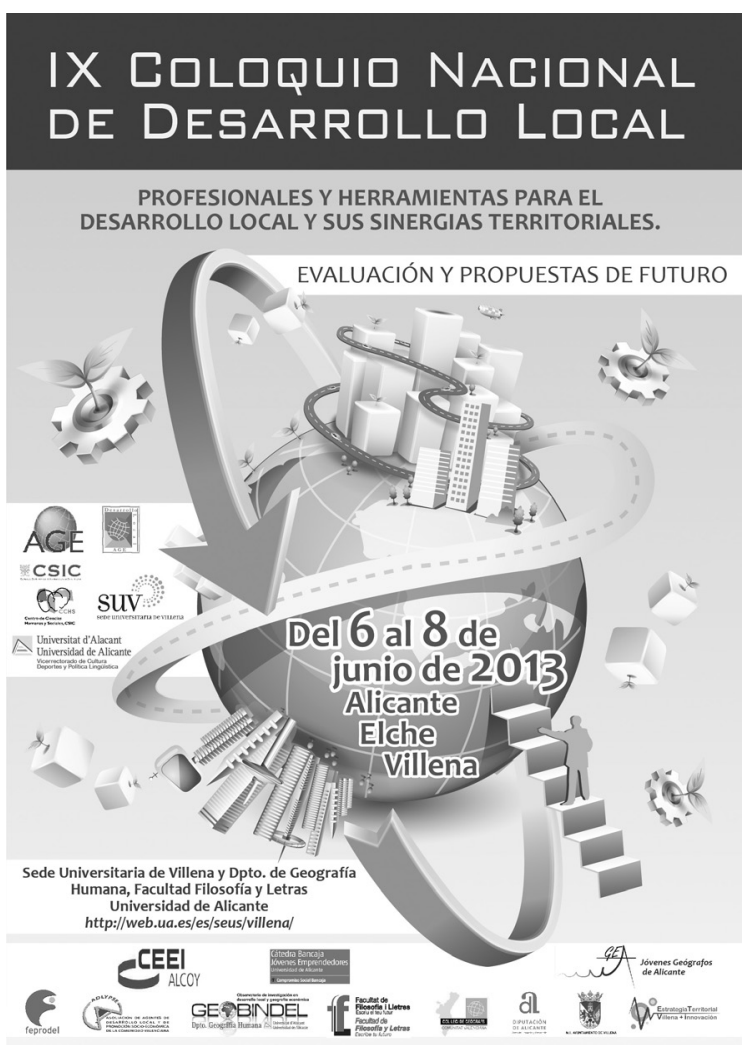


Este libro ha sido examinado y valorado por evaluadores ajenos a la Universidad de Alicante, con el fin de garantizar la calidad científica del mismo.

Publicacions de la Universitat d'Alacant

03690 Sant Vicent del Raspeig

Publicaciones@ua.es

http://publicaciones.ua.es

Telèfon: 965903480

(C) Antonio Martínez Puche, Xavier Amat Montesinos,

Isabel Sancho Carbonell y Daniel Sanchiz Castaño (eds.), 2016

(C) d'aquesta edició: Universitat d'Alacant

ISBN: 978-84-16724-00-0

Dipòsit legal: A 92-2016

Disseny de coberta: candela ink

Composició: Página Maestra (Miguel Ángel Sánchez Hernández)

Impressió i enquadernació: Guada Impresores

\section{unte \\ Unión de Editoriales
Universitarias Españolas \\ WWW.une.es
WWA}

Esta editorial es miembro de la UNE, cosa que garantiza la difusión y comercialización nacional y internacional de sus publicaciones.

Reservados todos los derechos. Cualquier forma de reproducción, distribución, comunicación pública o transformación de esta obra sólo puede ser realizada con la autorización de sus titulares, salvo excepción prevista por la ley. Diríjase a CEDRO (Centro Español de Derechos Repográficos, www.cedro.org) si necesita fotocopias o escanear algún fragmento de esta obra. 


\title{
MODELO DE REGIÓN SOCIALMENTE RESPONSABLE PARA EL DESARROLLO LOCAL: EL CASO DEL NORTE DEL CAUCA EN COLOMBIA Y DE LA COMUNITAT VALENCIANA EN ESPAÑA
}

\author{
Benjamín Betancourt \\ Director Grupo de Investigación en Previsión y Pensamiento Estratégico. \\ Universidad del Valle Colombia \\ benjabet@gmail.com \\ Johana Ciro Calderón \\ Coordinadora Línea de Investigación en Desarrollo Local e Innovación Social Estratégico. \\ Universidad del Valle Colombia \\ johanacirocalderon@gmail.com \\ Javier González Cardona \\ Presidente de la Federación Española de Profesionales de Desarrollo Local (FEPRODEL) \\ javigoncar@gmail.com
}

\section{RESUMEN}

"Región Socialmente Responsable - RSR" es una iniciativa de construcción para el desarrollo local en regiones específica a partir del ejercicio colectivo de la Responsabilidad Social. La Responsabilidad Social en tanto estrategia social y económica debe ser, ante todo, un asunto de conciencia y decisión ética que a la vez representa una oportunidad para el desarrollo empresarial que se traduce en competitividad y productividad, y en elemento de diferenciación si se asume como condición del funcionamiento de los mercados. El alcance del proceso fue impactar el entorno inmediato de las empresas del Norte del Cauca, en particular los municipios de Guachené, Caloto, Villarrica y Puerto Tejada en Colombia, y a su vez determinar los factores claves para aplicar dicho modelo en la Comunitat Valenciana.

Palabras clave: Cooperación, innovación social, desarrollo local, responsabilidad. 


\title{
MODEL OF SOCIALLY RESPONSIBLE REGION FOR THE LOCAL DEVELOPMENT: THE CASE OF THE NORTH OF CAUCA IN COLOMBIA AND OF VALENCIAN IN SPAIN
}

\begin{abstract}
RSR (Model of Socially Responsible Region), it is a construction initiative for the local development in specific regions starting from the collective exercise of the Social Responsibility. The Social Responsibility as long as social and economic strategy should be, above all, a matter of conscience and ethical decision that at the same time represents an opportunity for the managerial development that translates you in competitiveness and productivity, and in differentiation element if it is assumed as condition of the operation of the markets. The reach of the process was to impact the immediate environment of the companies of the North of Cauca, in particular the municipalities of Guachené, Caloto, Villarrica and Tiled Port in Colombia, and in turn to determine the key factors to apply this model in Valencia.
\end{abstract}

Key words: Cooperation, social innovation, I develop local, responsibility.

\section{INTRODUCCIÓN}

El enfoque de responsabilidad social se inspira en la propuesta del Pacto Global como iniciativa de compromiso ético destinada a que las empresas de todos los países acojan como una parte integral de su estrategia y de sus operaciones los 10 principios de conducta y acción en materia de derechos humanos, trabajo, medio ambiente y lucha contra la corrupción.

El primer hecho, las Regiones Socialmente Responsables como estrategia territorial de la Responsabilidad Social Empresarial (RSE) están siendo desarrolladas en distintos lugares de Colombia. En el caso particular del Norte del Cauca, la Región Socialmente Responsable surge como una iniciativa de los empresarios con el ánimo de dar respuesta a las necesidades locales y apostar a la construcción de un proceso de intervención social en el marco de una agenda regional de desarrollo, que incluya la perspectiva de múltiples actores como empresarios de las zonas francas asentadas en la zona, gobiernos locales, academia y representantes de la comunidad. Esta ponencia se propone describir el proceso de construcción colectiva de la Región Socialmente Responsable del Norte del Cauca para proponer su extracción y futura aplicación en la Comunitat Valenciana

En segundo hecho, En el caso particular de la Comunitat Valenciana, al igual que varias ciudades de España y Europa, se encuentra viviendo un momento determinante en términos de Innovación Social. El avance de este fenómeno a nivel global, asociado a la búsqueda de una sociedad más equitativa y 
de mayores oportunidades para todos, ha generado, entre otras cosas, el surgimiento de diversas iniciativas socioeconómicas en pos de estos objetivos y la reacción de múltiples actores sociales por dar cuenta de este fenómeno. Esta tendencia también ha tenido eco a nivel nacional, despertando un interés transversal por estas temáticas en la búsqueda del ansiado bienestar social. Particularmente, dentro del tercer sector y del sector privado, se han ido modificando las perspectivas tradicionales al incorporar nuevas dinámicas de innovación y emprendimiento social, capaces de generar valor social, y a su vez, contribuir a su propia sostenibilidad o competitividad financiera.

En este escenario, se debe mencionar que esta ponencia es fruto del trabajo investigación de los autores en el Norte del Cauca (Colombia), que ofrecen como insumo, entregar una base conceptual de manera que facilite la comprensión del análisis referido a la instalación de estas temáticas como instrumento para la Comunitat Valencia, para lo cual se revisa los principales términos, problemáticas y tendencias de este fenómeno a nivel global, para luego referirse al momento de oportunidad del contexto en la Comunitat Valenciana.

\section{LA RESPONSABILIDAD SOCIAL EN ESPAÑA}

"En un contexto de economía y sociedad global, caracterizado por una interdependencia cada vez mayor, el terreno de las consecuencias de nuestras acciones se amplía sensiblemente. Aumenta, por tanto, la responsabilidad de la empresa a la hora de tomar decisiones cuyas consecuencias afecten a los diferentes grupos de interés de la empresa (clientes, trabajadores, proveedores, sociedad y propietarios o accionistas)." (Fundación ETNOR, 2004: página 3).

En España, hasta el año 2003 el desarrollo de la RSE era todavía muy incipiente y embrionario. Aunque el debate público sobre la RSE llegó a España algunos años más tarde que a otros países europeos y tras la cumbre de Lisboa, a partir de 2003 el número de iniciativas promovidas por las empresas y las más diversas organizaciones e instituciones ha sido extraordinario. Ese desarrollo se ha dado además en la práctica totalidad de las Comunidades Autónomas. No obstante, habrá que esperar hasta el año 2007 para que la responsabilidad social de las empresas españolas tomase cuerpo como tema relevante del debate legislativo.

Veamos cómo se ha desarrollado su normativa en el cuadro I: 


\section{Cuadro 1. Normativa española}

\begin{tabular}{|c|c|}
\hline LEGISLACIÓN & CONTENIDO \\
\hline $\begin{array}{l}\text { Real Decreto 221/2008, de } \\
15 \text { de febrero }\end{array}$ & $\begin{array}{l}\text { Establece El Consejo Estatal de la Responsabilidad So- } \\
\text { cial de las Empresas (CERSE) órgano "asesor y consul- } \\
\text { tivo" del Gobierno adscrito al Ministerio de Empleo y } \\
\text { Seguridad Social y encargado del "impulso y fomento } \\
\text { de las políticas de Responsabilidad Social de las Em- } \\
\text { presas" en "el marco de referencia para el desarrollo de } \\
\text { esta materia en España" }\end{array}$ \\
\hline $\begin{array}{l}\text { Ley } 2 / 2011 \text {, de } 4 \text { de marzo, } \\
\text { de Economía Sostenible }\end{array}$ & $\begin{array}{l}\text { planteó nuevos retos a dicho Consejo, por la aproba- } \\
\text { ción del artículo } 35 \text { referente a las obligaciones para el } \\
\text { sector público empresarial; y el artículo } 39 \text { en lo que se } \\
\text { refiere a la promoción de la responsabilidad social de } \\
\text { las empresas }\end{array}$ \\
\hline $\begin{array}{l}\text { La Ley 50/2002 de Funda- } \\
\text { ciones }\end{array}$ & $\begin{array}{l}\text { La regulación de la responsabilidad social solidaria. } \\
\text { Las Fundaciones, el patrocinio y el mecenazgo. }\end{array}$ \\
\hline \multicolumn{2}{|l|}{ Comunitat Valenciana } \\
\hline $\begin{array}{l}\text { La Ley } 11 / 2009 \text {, de } 20 \text { de } \\
\text { noviembre, de la Generali- } \\
\text { tat, de Ciudadanía Corpo- } \\
\text { rativa }\end{array}$ & $\begin{array}{l}\text { Pretende poner los medios para que las empresas y } \\
\text { personas profesionales autónomas interesadas puedan } \\
\text { desarrollar los sistemas, programas y acciones que con- } \\
\text { sideren oportunos. Y lo hace a través de dos herramien- } \\
\text { tas fundamentalmente: la certificación de la excelencia } \\
\text { en la ciudadanía corporativa y, a partir de estas certifi- } \\
\text { caciones, anualmente, la elaboración de un Índice de } \\
\text { Ciudadanía Corporativa como medida de fomento com- } \\
\text { plementaria. Esta ley debería ser modificada en aras de } \\
\text { la unidad de mercado. }\end{array}$ \\
\hline
\end{tabular}

Fuente: Elaboración Propia CIRO 2014.

Sin embargo, España presenta un considerable retraso respecto a los países europeos. Es cierto que, durante los últimos años, se ha observado que las empresas españolas han ampliado el contenido de RSE en sus memorias e informes, lo cual constata una evolución positiva al respecto, pero con significativas diferencias entre empresas, y todavía muy alejada de los niveles alcanzados en otros países europeos.

En este sentido, una de las impotencias del modelo de RSE en España es el restringido diálogo normalizado entre las empresas y la sociedad civil que se manifiesta en los iniciales procesos y políticas de RSE aplicadas. También se evidencia cómo se ha producido una confusión generalizada en la opinión pública entre la RSE y la acción social o marketing social, que en absoluto ha ayudado a gestionar adecuadamente la RSE. Además, otra carencia también ob- 
servada en el mercado español es que, usualmente, las empresas no desarrollan estrategias de RSE vinculadas a sus actividades y negocios clave, sino que se plantean como un área adyacente a la gestión de la empresa, entrando en altos riesgos de discrepancias con la gestión.

Por tanto, es cierto que en España cada vez más organismos están impulsando iniciativas de diversa índole en el campo de la RSE, pero queda mucho camino por recorrer para ampliar el conocimiento de las acciones emprendidas y extender la cultura de la RSE.

No obstante, hasta la fecha, tan sólo se han aplicado de forma parcial el lenguaje, conocimientos y herramientas de la teoría financiera de la empresa en la orientación de los procesos de decisión de los diferentes agentes económicos de cara a incorporar objetivos de RSE.

Por tanto, en España, es especialmente significativo el escaso desarrollo de los instrumentos de medición de la RSE, la dimensión parcial de algunos y el bajo nivel de implantación de la RSE. No se observa una relación entre rentabilidad económico-financiera con una mayor información de carácter social o medioambiental.

Ahora bien, en todas las modalidades de Responsabilidad Social, la asunción de las mismas por parte de las empresas se halla directamente relacionada con la dimensión empresarial atendiendo tanto a facturación como a número de empleados. Así, "el 40,5\% de las empresas Socialmente responsables realizan donaciones en especie (productos y servicios); el 24\% del total de las empresas de la Comunidad Valenciana. En números absolutos un total de 46.081 empresas valencianas realizan donaciones en especie a entidades cívicas y sociales ${ }^{1 "}$.

Por todo lo anterior, es necesario hacer una llamada a las instituciones públicas con responsabilidad en este campo, porque se hace necesario llevar a cabo políticas de difusión que contribuyan al conocimiento de esta nueva cultura empresarial que beneficia tanto a la comunidad, como a las empresas y a los trabajadores/as. Un nuevo modelo de región socialmente responsable, superando la clásica concepción de la empresa como organización que sólo busca un beneficio económico y vinculándola al territorio.

Por todo lo anterior, consideramos que es el momento propicio para abordar otras iniciativas en materia de RSE, de ahí que proponemos el modelo de regiones socialmente responsable desarrollado en Colombia, como un insumo de reflexión que invite a replantear la RSE no sólo desde la empresa sino como una estrategia territorial que reúne valioso instrumentos en desarrollo local.

1 IMPACTO ECONÓMICO Y SOCIAL DE LA RESPONSABILIDAD SOCIAL EMPRESARIAL EN LA COMUNIDAD VALENCIANA (pp. 113-141) - CIRIEC. 


\section{Modelo De Región SOCIALMENTE RESPONSABLE}

Sostenibilidad de la Región a partir de la Responsabilidad Social y la Interacción de las tres dimensiones para maximizar su eficiencia la RS busca desplegarse a través de mecanismos colectivos asociados al funcionamiento económico. Los principales son: sectoriales, cadena de valor, temáticos y territoriales.

Las iniciativas sectoriales son de carácter de auto-regulación por parte de algún sector global de la producción o del funcionamiento económico que mediante el establecimiento de códigos y estándares de conducta busca que todas las empresas inscritas en dicho sector alcancen un comportamiento ético de acuerdo a lo definido. Las iniciativas de mayor fuerza en la actualidad son las del sistema financiero global, el sector extractivo, la agroindustria y la construcción.

En cualquiera de las dos situaciones el propósito es lograr que el conjunto de personas, instituciones y empresas que convergen en un propósito productivo o de prestación de servicios orienten sus actuaciones bajo un acuerdo ético compartido. Al igual que las sectoriales las de cadena de valor se consideran iniciativas de autorregulación.

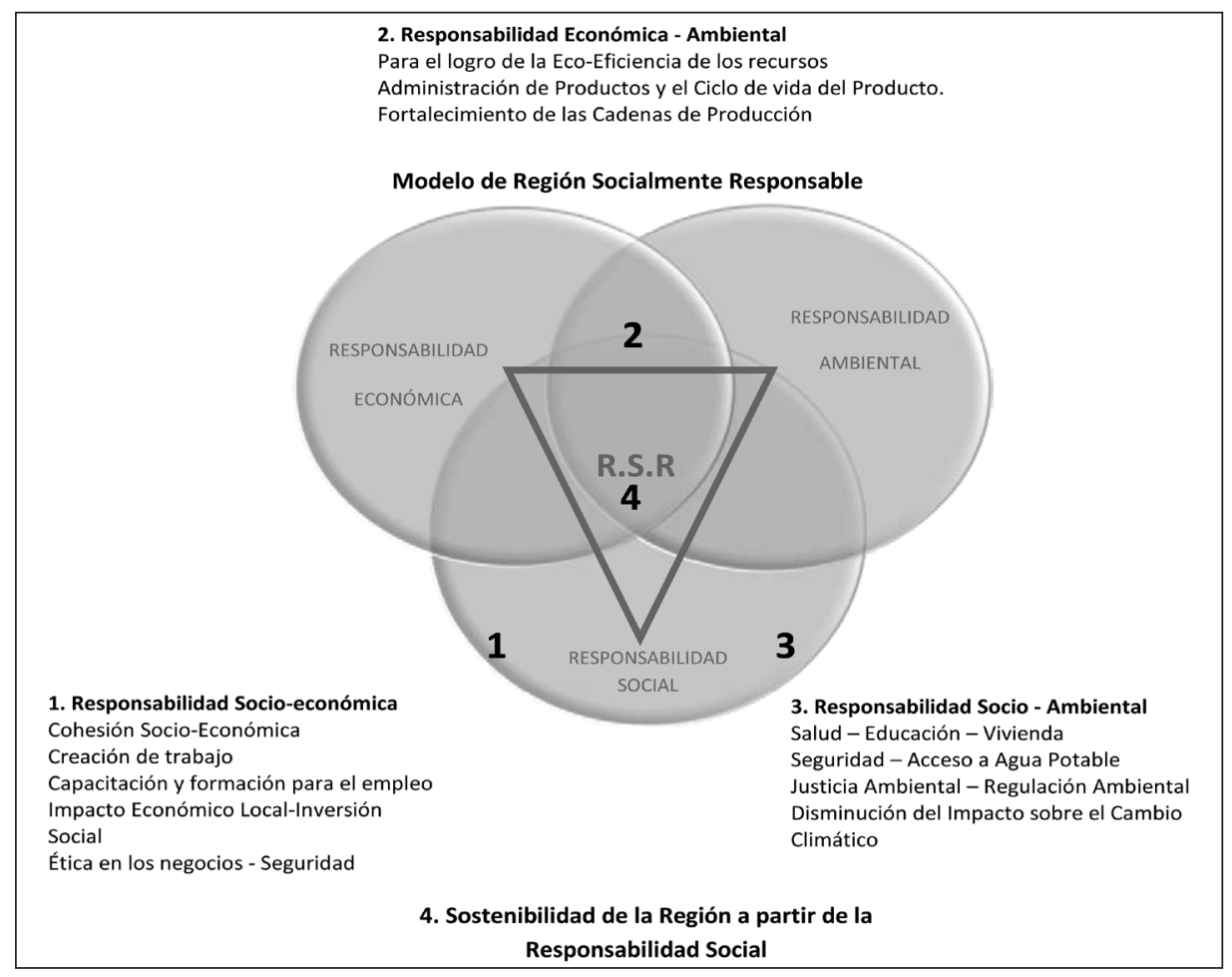

Figura 1. Modelo de Región Socialmente Responsable.

Fuente: Betancourt, B. 2010. 
Las iniciativas temáticas se enfocan en garantizar el comportamiento social y empresarial en algún aspecto específico de las dimensiones del desarrollo de situaciones sociales críticas. Las hay de origen de auto regulación como son el "Mandato por el Agua" y "El Cuidado del Clima" impulsadas por el Pacto Global de Naciones Unidas y las hay de carácter de Control Social orientadas por organizaciones de la sociedad civil global que buscan impulsar actitudes y prácticas sociales y empresariales que protejan derechos ciudadanos. En materia de Derechos Humanos es en donde más iniciativas de este tipo de encuentran y se relacionan con asuntos tales como la abolición del trabajo infantil, la discriminación en los entornos de trabajo, la protección de derechos laborales, de la biodiversidad, etc.

Y finalmente las iniciativas territoriales (Regiones Responsables) parten de la consideración de una región como un escenario local para el despliegue del comportamiento social general y buscan que dicha región o territorio, como un todo, logre establecer acuerdos fundamentales de comunidad de propósito colectivo y arreglos de funcionamiento social que permitan el desarrollo humano y la sostenibilidad.

Estas iniciativas si bien pueden tener impulso en cualquiera de los actores de la sociedad, se orientan a activar el marco social de tres componentes: regulación del Estado, auto regulación empresarial y control de la sociedad en general, en una única perspectiva de convergencia dentro de un territorio.

Cualquiera que sea la iniciativa, todas apuntan en esencia a lo mismo: a lograr transformaciones en la comprensión social de nuestro devenir y por tanto cambiar comportamientos, actitudes y prácticas sociales de manera que pueda construirse desarrollo humano y sostenibilidad en la sociedad.

El programa de Regiones Responsables fue creado en Colombia en 2007 como una iniciativa de la Red del Pacto Global con el ánimo de ofrecer mecanismos prácticos colectivos para el ejercicio de la Responsabilidad Social. En la actualidad "Regiones Responsables" es un programa autónomo, con organización propia, con el apoyo del Pacto Global.

Una "Región Responsable" se sustenta en la noción de "Sistema Social" que se configura en una región o territorio específico y en el que se desenvuelve la vida social, política, económica, cultural y espiritual de una comunidad. En tanto región se entiende como un sistema interdependiente entre sí y con el entorno natural que configura un interés común que se garantiza a través de un Contrato Social".

El contrato social de una Región Responsable se enfoca, en principio, en cuatro elementos básicos:

1. Ciudadanía responsable: activación del ejercicio cívico de los ciudadanos que asumen sus responsabilidades frente al colectivo y ejercen de manera activa y constructiva sus deberes y derechos. 
2. Economía responsable: funcionamiento económico basado en la ética, la justicia social, la cohesión social y la equidad, se reducen los impactos y se agrega valor colectivo expresado en desarrollo humano y sostenibilidad.

3. Educación para la responsabilidad: formación de líderes y ciudadanos capaces de conducir a la sociedad hacia el rumbo del desarrollo humano y la sostenibilidad.

4. Política responsable: ejercicio transparente, participativo y eficaz de la política en un modelo democrático de múltiples niveles de gobernabilidad del desarrollo.

Una Región Responsable es aquella que logra identificar los factores objetivos y subjetivos del colectivo que favorecen o impiden el logro de propósitos de desarrollo y los transforma a partir de la conciencia de la responsabilidad social y a través de modelos público privados de movilización y participación ciudadana.

A nivel conceptual "Regiones Responsables" integra los planteamientos del desarrollo local, de la gobernabilidad pluralista y de múltiples niveles, y por supuesto, de la Responsabilidad Social.

\section{Experiencia práctica: Región Socialmente Responsable en el Norte del CAUCA}

Región Socialmente Responsable en el Norte del Cauca es una propuesta para construir de manera colectiva un modelo de desarrollo para el Norte del Cauca, teniendo como nodos articuladores los municipios de Puerto Tejada, Villarrica, Guachené y Caloto, que propicie un cambio en las condiciones sociales, económicas y ambientales que son dimensiones clave en el desarrollo de esta región, que históricamente ha tenido grandes retos con relación a sus indicadores de desarrollo, a la vez que tiene grandes potencialidades observadas en el crecimiento económico de la última década, propiciado por la Ley Páez (Ley 218 de 1995).

Este proyecto que se desarrolló en el marco del Convenio No. 163 -2007 suscrito entre Colciencias y la Universidad del Valle a través del Instituto de Prospectiva, Innovación y Gestión del Conocimiento, es un espacio de articulación de la Universidad con los empresarios del Norte del Cauca que preocupados por la situación de las comunidades, proponen la construcción de una agenda regional de desarrollo, que tiene como fundamento la responsabilidad social empresarial. Adicionalmente, en un ejercicio pionero para estos temas, se incluye la metodología prospectiva, para darle sostenibilidad a un proyecto de esta magnitud.

La presente investigación recoge no sólo los resultados del proyecto, sino también la aplicación de metodologías prospectivas para la realización de los 
talleres con los actores sociales de la región, gracias a lo cual se pudo analizar de conjunto la realidad del Norte del Cauca y las capacidades regionales para construir un plan de futuro.

El enfoque de responsabilidad social se inspira en la propuesta del Pacto Global como iniciativa de compromiso ético destinada a que las empresas de todos los países acojan como una parte integral de su estrategia y de sus operaciones los 10 principios de conducta y acción en materia de derechos humanos, trabajo, medio ambiente y lucha contra la corrupción.

Este es un estudio centrado en el ser humano. La pertinencia de esta investigación, es que recoge las voces de los actores como los niños, los jóvenes, las instituciones, los gobiernos locales, los empresarios, la academia, la comunidad en general, entre otros. Se analiza de manera profunda la realidad de la región y se identifica de forma colectiva las propuestas para atender las necesidades y superar los problemas que la afectan, construyendo escenarios de futuro posibles donde se incorporen criterios de inclusión, equidad y desarrollo de todos los actores.

\section{Metodología}

Objetivos de Investigación

- Ante los retos y desafíos que ha impuesto el establecimiento del Parque Industrial y Comercial en la zona norte del Departamento del Cauca derivado de la Ley Páez y con un panorama de permanencia a largo plazo al convertir el Parque Industrial en Zona Franca, un grupo de empresarios e instituciones interesados por el desarrollo de la región inició desde el 2008 un acercamiento con la Oficina del Global Compact en Colombia, y ha logrado adelantar un proceso de diálogo entre los diversos actores regionales para el desarrollo integral bajo el modelo de región socialmente responsable.

- En este contexto, y con la intención de consolidar estos esfuerzos se ha formulado este proyecto con el objetivo de articular a los diversos actores públicos y privados de la Región Norte del Cauca en una estrategia de Región Socialmente Responsable - RSR, para lograr el desarrollo integral de las empresas, las instituciones, los municipios y las comunidades de los municipios de Caloto, Guachené, Puerto Tejada y Villarrica. El eje central de la agenda es la Responsabilidad Social Empresarial en el marco de una estrategia de región por medio del cumplimiento de los objetivos en 5 fases así:

- FASE 1: Fundamentación de la acción.

- FASE 2: Capacidades empresariales y capacidades sociales instaladas.

- FASE 3: Escenarios de realidades y futuros

- FASE 4: Construcción de acuerdos

- FASE 5: Preparación transversal 


\subsection{Fases del proceso}

En el proyecto se aplican metodologías prospectivas para analizar de conjunto la realidad económica, social y las capacidades regionales para construir un plan de futuro. Se combinaron métodos cuantitativos y cualitativos y se adelantó un proceso de consulta con los actores sociales de la región para el diseño y puesta en marcha del proceso de construcción que sirva de plataforma para la formación de capacidades endógenas de responsabilidad social para el desarrollo regional.

La caracterización de región es uno de los pilares fundamentales del proyecto seguido del ejercicio de prospectiva, el diseño de programas, proyectos y acciones y finalmente su socialización y compromisos de los actores.

Es un ejercicio de planeación participativa en cuatro fases: Diagnóstica y de Caracterización de la Región; Prospectiva y Estrategia; Operativa y Socialización, en todas las cuales están presentes los actores sociales.

Los elementos temáticos que alimentan el modelo de RSR son los siguientes: Dimensión Económica, Dimensión Socio-Cultural y la Dimensión Ambiental en un entorno donde la intervención del estado y la participación de las instituciones involucradas contribuyen al mejoramiento de la región.

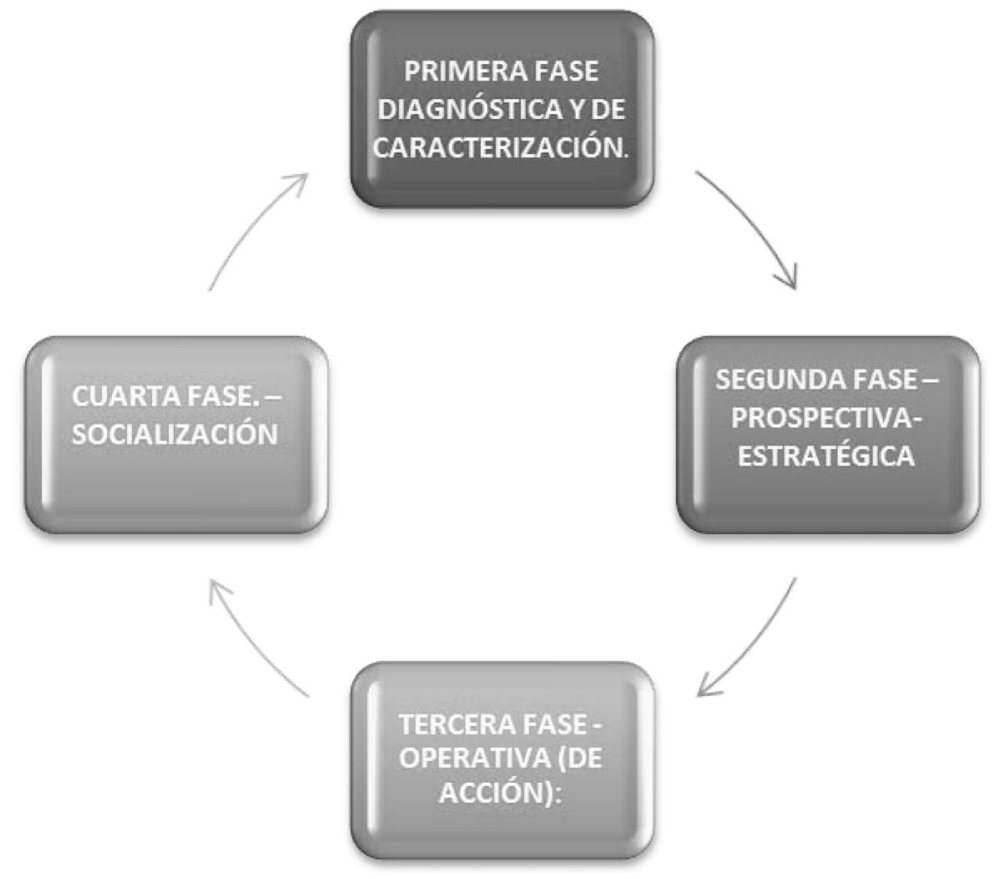

Figura 2. Elementos temáticos del modelo de RSR.

Fuente: elaboración propia. 
5.2. Análisis comparativo de la RSE en la Comunitat Valenciana (España) Vs El Norte del Cauca (Colombia)

El escenario de la RSE tanto en Colombia como en España o cualquier otra región del mundo, está estipulada por el entorno en que opera la empresa. Hay una serie de condiciones con influencia determinante en el comportamiento empresarial.

No tenemos conocimiento de estudios donde se comparen las acciones de RSE entre la Comunitat Valenciana en España y el Norte del Cauca en Colombia. Sin embargo, la situación de la RSE en las Pymes en el Norte del Cauca y en la Comunitat Valencia por separado puede servir, aunque con limitaciones, para hacer algunas comparaciones. El estudio comparativo fue realizado con una parte del cuestionario que contiene preguntas semejantes y fue diseñado por el mismo equipo que llevó a cabo el estudio de Regiones Socialmente responsables en Colombia. Si bien las Pymes no son indicativas de las prácticas de la gran empresa, la comparación puede dar luces sobre el papel diferente que pueden jugar las actividades responsables de las empresas. El estudio cubrió actividades de responsabilidad interna, vale decir sobre el personal, gobierno corporativo, proveedores, el consumo de recursos (agua, electricidad, reciclaje, subproductos, etc...), actividades de responsabilidad externa (hacia la comunidad) y actividades de responsabilidad medio ambiental.

De los resultados de esta comparación se observa que hay más actividad de responsabilidad social empresarial externa en las PYME del Norte del Cauca que en la Comunitat Valenciana. Sin entrar en detalles, podemos por ejemplo analizar las razones por las cuales la empresa es responsable. En la Comunitat Valenciana las principales razones aducidas para ser socialmente responsable con la comunidad, son, mayoritariamente, de tipo ético-religioso También en Colombia son la principal razón para ser responsable ante la comunidad, pero con menor intensidad que en Comunitat Valenciana. En el Norte del Cauca la presión de la comunidad es un poco mayor, probablemente porque hay una mayor identificación de las comunidades con la misma empresa. Las presiones de los empleados, socios y clientes juegan un papel un poco más relevante en el Norte del Cauca que en la Comunitat Valencia, aunque en general las razones no son muy distintas.

En cuanto a las actividades de apoyo, en el Norte del Cauca se enfatiza mucho más el apoyo a la educación y a colectivos desfavorecidos, como discapacitados, las poblaciones indígenas o afro-descendientes. Ello puede explicarse por los condicionantes que mencionábamos anteriormente: porque las necesidades sociales y ambientales son más evidentes y es necesario cubrir deficiencias en la actuación del sector público. En la Comunitat Valenciana las empresas Pymes dedican muchos más recursos a actividades deportivas, en ambos casos se dedican más o menos los mismos recursos en salud, cultura y 
medioambiente. En cuanto a la forma, en el Norte del Cauca hace mucho más vía donaciones directas, y con más participación de empleados y gerentes, y menos vía patrocinio, que es el esquema más favorecido en la Comunitat Valenciana.

En términos generales se puede concluir diciendo que en la Comunitat Valenciana se hace más por motivos de mayor eficiencia y mejora de imagen. Con esto no quiere decir que en Comunitat Valenciana no se hace para cumplir con la legislación vigente, sino que en el Norte del Cauca se hace porque la ley lo pide, y menos por convencimiento. En el Norte del Cauca hay que forzar la actividad en RSE, a través de legislación. Eso también nos da algún indicador para el diseño de política pública. En algunos casos no hace falta tener una política pública, porque el comportamiento es espontáneo, pero en otras hay que forzar ese comportamiento.

Las razones primordiales por las cuales no se realizan prácticas de responsabilidad social en la Pyme de la Comunitat Valenciana es por falta de tiempo, en el Norte del Cauca es por falta de recursos y por falta de conocimiento de los beneficios que la responsabilidad puede traer.

\section{Cuadro 2. Tabla resumen problemas}

\begin{tabular}{|l|l|}
\hline \multicolumn{1}{|c|}{ Comunitat Valenciana (España) } & \multicolumn{1}{|c|}{ Norte del Cauca (Colombia) } \\
\hline - Intensivas en consumo, necesidad de aho- & - Tratando de sobrevivir \\
rro, reciclaje & - Economías emergentes \\
- Economías maduras & - Amplio territorio, menos necesidad de \\
- Dependencia de materias primas de otros & conservar \\
- Políticas regionales (salud, bienestar, me- & - Políticas inestables, y en sostenibilidad, \\
dio ambiente, laboral, etc.) & pobres o limitadas \\
- Presión al buen gobierno & - Ineficiencia es tolerada, corrupción existe \\
- Influencia de las partes interesadas & - Subdesarrollo de partes interesadas \\
- "Competencia" entre países & (stakeholders) en la RSE \\
- Buena infraestructura, no es un limitante & - Cada uno por su cuenta \\
- Presión a gobiernos para demostrar y for- & - Bastante preocupación hay con resolver \\
zar sostenibilidad & problemas cotidianos \\
- Globalización natural & - Sostenibilidad no es un lujo, pero no es \\
- Altos ingresos en algunos sectores & prioridad \\
- Recursos humanos capacitados & - Aislamiento de algunos sectores, mirando \\
- Promoción RSE en el ámbito de toda Eu- & hacia adentro \\
ropa que favorece a la Comunitat Valen- & - Desigualdad en la población es un proble- \\
ciana & ma (condiciona tipo \\
- Servicios públicos relativamente amplios & - de consumo) \\
y eficientes & - Desconfianza de casi todos \\
- Empresa cerca del gobierno & \\
\hline
\end{tabular}

Fuente: Elaboración propia, 


\section{Conclusiones}

Este artículo inicia su aporte sobre la metodología y prospectiva y estratégica aplicada a región Socialmente responsable Norte del Cauca, realiza una reflexión sobre un proceso social y regional concreto, a partir de mi participación en el mismo como investigador y coordinador metodológico. En el proceso investigativo de RSR- Norte del Cauca, los actores sociales de la región y el equipo de investigación del proyecto trabajaron conjuntamente para formular y poner en práctica el modelo de región socialmente responsable en el Norte del Cauca. El proyecto se formuló abarcando cuatro localidades: Guachené, Puerto Tejada, Villarrica y Caloto.

Se logró desarrollar los objetivos del proyecto y establecer con los actores sociales de la región, así como las localidades vinculadas al estudio, una visión de región, avanzando en el diseño de un modelo de responsabilidad social en las cuatro localidades objeto de estudio para formación de capacidades endógenas de esta manera conseguir el desarrollo local.

Este es un artículo de planificación prospectiva y estratégica del territorio, que aporta el conocimiento necesario sobre la problemática específica de la región nortecaucana y ayuda a situarla en contexto, facilitar la toma de decisiones de sus líderes al reducir la incertidumbre y el riesgo derivado del desconocimiento o la intuición.

En todas las Comunidades Autónomas de España tienen presión hacia el buen gobierno corporativo. En Colombia lamentablemente la ineficiencia es tolerada, se ha aprendido a vivir con ella, a coexistir con esa ineficiencia y aun cuando España no está exenta de corrupción, en Colombia, lamentablemente, está más generalizada.

En la Comunitat Valenciana se percibe una gran influencia de las partes afectadas, en contraste, el Norte del Cauca hay un gran subdesarrollo de esas partes interesadas.

En la Comunitat Valenciana se compite entre comunidades autónomas para subir el listón, en el Norte del Cauca cada departamento va por su cuenta.

En Comunitat Valenciana tienen una buena infraestructura, no es un factor limitante, en el Norte del Cauca hay ya bastante con la preocupación de resolver problemas.

En la Comunitat Valenciana se presiona a los gobiernos para demostrar y forzar la sostenibilidad. En el Norte del Cauca la sostenibilidad no es un lujo, pero tampoco es una prioridad.

En la Comunitat Valenciana se ve la globalización más o menos de manera natural, en el Norte del Cauca hay muchos sectores que todavía miran sólo hacia dentro.

En la Comunitat Valenciana hay altos ingresos, en el Norte del Cauca la desigualdad es un gran problema. Y aquí hay un condicionante muy claro sobre 
la diferencia en el papel que debe jugar la responsabilidad social. La desigualdad es un problema, y resolverlo, aun cuando no le competa a la empresa, sí debe ser su preocupación y debe condicionar sus actuaciones.

Los servicios públicos en la Comunitat Valenciana son relativamente amplios y eficientes, pero en el Norte del Cauca hay un fallo del Estado en la provisión de los servicios, que en algunos casos es también sumamente crítico y que también condiciona la actuación de la empresa. En algunos casos se piensa que la empresa tiene la responsabilidad de resolver este fallo y aunque estrictamente hablando no es responsabilidad de la empresa, se puede ver forzada a buscar la solución. La empresa en la Comunitat Valenciana está "cerca" del gobierno. En algunas ciudades del Norte del Cauca la empresa está "dentro" del gobierno Por último, esta comunicación propone socializar los resultados alcanzados y ponerlos a consideración de los actores sociales para incentivar un diálogo que beneficie la adaptación de este modelo y su posterior aplicación en la Comunitat Valenciana, dado que esta tiene factores de avance mayores que el Norte del Cauca, por lo cual se pude decir que si los resultados de la aplicación del modelo en el Norte del Cauca han sido tan fructíferos partiendo de un alto grado de incertidumbre, las posibilidades de éxito de este modelo en la Comunitat Valenciana pueden ser altas.

BIBLIOGRAFÍA

ALBURQUERQUE F., Desarrollo económico territorial. Guía para agentes, Sevilla: Instituto de Desarrollo Regional, Fundación Universitaria, 2002.

ALIANZA COOPERATIVA INTERNACIONAL (ACI), "Los principios cooperativos del siglo XXI", CIRIEC-España. Revista de debate sobre la economía pública, social y cooperativa, $\mathrm{n}^{\circ} 19,1995$, pp. 38-39.

BAREA, J. y MONZÓN, J. L. (directores), Libro Blanco de la Economía Social en España. Madrid: Ministerio de Trabajo y Seguridad Social, 1992.

BAREA, J. y MONZÓN, J. L. (directores), Informe sobre la situación de las cooperativas y las sociedades laborales en España. Valencia: Editorial CIRIEC-España, 1996.

BARREIRO, F., "El modelo de Desarrollo Local y el papel de la Economía Social: un enfoque en torno a los NYE", Economía Social, Nuevos Yacimientos de Empleo y Desarrollo Local, 2002.

BUENDÍA, I y VIDAL, J., Cooperativismo y desarrollo local. Madrid: Centro de estudios Ramón Areces, Fundación Luís Vives, 2003.

CACHÓN, L., "Los nuevos yacimientos de empleo en España: una (primera) visión general", Revista Economía y sociología del trabajo, no 29-30, (Ejemplar dedicado a: Nuevas necesidades, nuevas actividades y nuevos empleos: desafíos y perspectivas), 1996, pp. 124-142.

CALVO, R. y LERMA, I., "Una aproximación a las relaciones laborales-contractuales generadas por una agencia de desarrollo local (ADL): propuesta 
de clasificación y conclusiones críticas", Revista Documentación laboral, $n^{\circ} 80,2007$, pp. 145-155.

CHAVES, R. y SAJARDO, A., "La inserción laboral a través de la economía social en el ámbito de las políticas activas de empleo", Revista Valenciana d'Estudis Autonòmics, $\mathrm{n}^{\circ}$ 26, 1999, pp. 57-84.

COQUE, J., "Industrialización en el entorno local a través de sociedades cooperativas", REVESCO Revista de Estudios Cooperativos $n^{\circ}$ 68, (Ejemplar dedicado a: Jornadas técnicas sobre cooperativismo y desarrollo local), 1999, pp. 119-137.

DIPUTACIÓN DE ALICANTE, Manual para la creación y funcionamiento de una Agencia de Desarrollo Local. Alicante, 2000.

GARCÍA-GUTIÉRREZ, C., "Cooperativismo y desarrollo local”. REVESCO Revista de Estudios Cooperativos $\mathrm{n}^{\circ}$ 68, (Ejemplar dedicado a: Jornadas técnicas sobre cooperativismo y desarrollo local), 1999, pp. 33-46.

GÓMEZ, J.M. y ROMÁN, A., "La economía social y su contribución a la promoción del desarrollo local y regional", Revista Universitaria de Ciencias del Trabajo, $\mathrm{n}^{\circ}$ 6, 2005, pp. 254-289.

MADOERY, O., "El proyecto local como alternativa de desarrollo", en Fernández, A y Gaveglio, S. Globalización, Integración, Mercosur y Desarrollo Local. Rosario (Argentina): Homo Sapiens, 2000.

MORÓN, P., "Los agentes de la Economía Social coordinadores y gestores de las políticas públicas: aplicación a la integración socio-laboral de jóvenes marginados”, CIRIEC - España. Revista de economía pública, social y cooperativa, $\mathrm{n}^{\mathrm{o}} 24,1996$, pp. 63-90.

SANCHÍS, J. R., Dirección Estratégica de Empresas Cooperativas, Valencia: Promolibro, 2001. 